\title{
Development of a system of transport-interchange hubs in urban transport network
}

\author{
Oleg V. Moskvichev ${ }^{1}$, and Svetlana A. Leonova ${ }^{1, *}$ \\ ${ }^{1}$ Samara State Transport University, 18, Zavodskoy highway, Samara, Russia
}

\begin{abstract}
The paper considers the issues of the formation of a system of transport interchange hubs (TIH), associated with the determination of the location of TIH in order to improve the quality of the provided transport services. The model of the optimal choice of TIH system is presented in the form of a linear Boolean programming problem. The paper gives the factors that reduce the average travel time around the city due to the formation of a system of transport hubs. In order to solve the problem of the choice of TIH locations, the approach based on the definition of "effective interchange hubs" is proposed. The determination of effective interchange hubs is carried out according to the following algorithm. First we found a list of non-stop (direct) rides. Then, we consider the rides for which there is no direct route or for which there is such a route, but this revealed connecting route will take less time. These hubs are efficient. Next, a list of effective interchange hubs is determined and the amount of passenger traffic at each hub is specified. Using mathematical methods to solve integer programming problems, it is possible to select such efficient interchange hubs, in which it is advisable to create transport interchange hubs. These scientific results can be used in programs and projects for the development of urban transport systems in the development of basic requirements for the formation of transport hubs.
\end{abstract}

Keywords: transport interchange hub, urban transport network, efficient interchange hub, optimal choice, linear Boolean programming problem, passenger traffic

\section{Introduction}

Recently, the problem of the development of urban transport infrastructure in cities and megalopolises, including transport interchange hubs (TIH), has become increasingly important. A special place in the formation of a modern urban transport system is assigned to transport and interchange hubs. Urban transport hubs are formed at the intersection of various types of public passenger transport. The number of transport hubs and their locations determine the state of the entire transport system of urban public transport. Optimal placement of TIH increases the attractiveness of public urban transport ensuring fast, safe and comfortable travel through interchange hubs.

In the scientific literature, the choice of the locations of TIH in the network of urban public transport is based on the use of the expert assessment method $[11,14]$. It is also proposed to create transport and interchange hubs on the basis of railway stations, passenger depots and metro stations. According to this principle, transport hubs have been formed in super-large cities of Russia $[3,11,12,14,15]$ and foreign countries $[5,10]$.

However, in many cities, high-speed off-street transport (urban rail transport, subway) is insufficiently developed, which must also be taken into account when designing additional transport infrastructure, including
TIH. It is necessary to conduct a comprehensive analysis of the state of the road network and the demand for urban public transport based on the construction of quantitative models. The important characteristic of a transport hub is the amount of passenger traffic.

In order to solve the problem of the choice of the location of TIH, it is necessary to select the type of model. The analysis of the literature on the development and use of mathematical models for the description of urban passenger transport processes $[1,3,8,9,12,13$, 15] shows that most of them use a simulation tool. For example, N.A. Kalyuzhny [2, 3] considers the VISUM software package and Citraf software package, which the author chose because of the simplicity and availability of modification. The use of this tool allowed identifying some properties of the network of urban passenger transport, which indirectly indicate those network hubs that can become TIHs. In this case, only the hubs formed around the stations of high-speed off-street transport are considered.

The criterion of the influence of the waiting time delay revealed by the author can be considered only as one of the factors determining the priority of the construction of the transport-interchange hub, but there are a number of other factors that can influence the choice of the location of the transport-interchange hubs. It is necessary to note that the conclusions drawn on the

Corresponding author: moskvichev063@yandex.ru 
basis of simulation modeling of the urban transport system are not universal in nature and it is incorrect to apply them for the network of any city. Moreover, the network parameters that have to be pre-entered into the model require large statistical measurements of passenger traffic in a particular network. As a result, further scientific research and substantiation of the issues of the choice of the location of urban transport hubs in order to improve the urban transport system require further study.

\section{Problem Statement}

For the accurate assessment of the state of the urban transport system and its development, including the development of urban transport and interchange hubs, it is required to perform a system analysis of the processes occurring in the urban passenger network, based on criteria expressing the main purposes of the system and build models that express the target function as the degree of achievement of the main purpose.

Therefore, in order to characterize the quality of the urban transport system, the value of the average statistical time of one ride is taken. The optimal location of urban transport hubs in order to improve the quality of the provided transport services is formally expressed as an objective function

$$
\bar{T}=f(X) \rightarrow \min ,
$$

where $\mathrm{X}$ denotes a certain variable subset of $\mathrm{TIH}$, which is a variant of constructing a TIH system for the entire urban passenger network.

It is obvious that in the general case, during the choice of the optimal system (set) of $\mathrm{TIH}$, there are always restrictions on the choice of $\mathrm{X}$. Therefore, the options of $\mathrm{X}$ must belong to some admissible set $\mathrm{G}$, that is, $\mathrm{X} \in \mathrm{G}$. Thus, in general form, the problem of optimal choice of the TIH system can be written as

$$
\begin{aligned}
& \bar{T}=f(X) \rightarrow \min \\
& X \in G
\end{aligned}
$$

Consequently, the simulation of the processes occurring in urban passenger transport should describe the objective function (1) and explicitly express the constraints (2). The urban public transport network is a graph that includes subgraphs of all types of urban public transport and a subgraph of transfers. The network graph includes stops and routes of urban public transport (Figure 1).

The connectivity of the graph is taken into account during the solution of the problem of the choice of the location of TIH. It is known that not all dependencies between the parameters of the graph model can be described in the form of analytical dependencies. In addition, we should expect a model with an extremely large number of variables and constraints, which makes the search for a solution labor-intensive or almost impossible. As a result, it is important to propose an approach that will allow solving the problem of the optimal choice of locations for urban transport hubs.

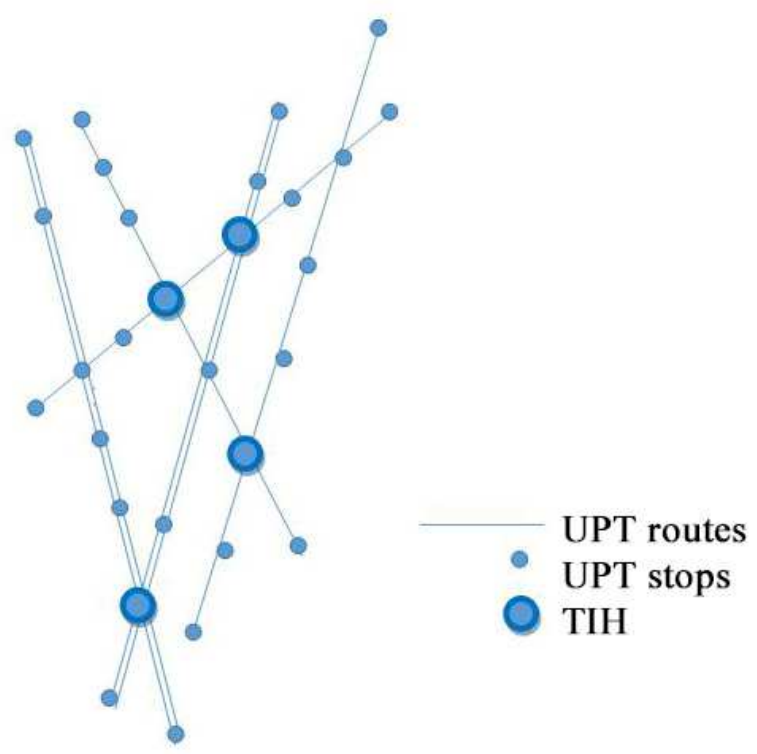

Figure 01. Network graph of urban public transport (UPT)

\section{Model of the optimal choice of the TIH system in the form of a mathematical programming problem}

In order to solve the problem of optimal choice of the location of transport hubs for urban transport network, a possible mathematical model should be considered. The network of urban public transport is a graph with stops and routes of urban public transport (Figure 1). In the first assumption it is considered that any passenger route within a straight line is the best and no choice is required. If the starting and ending stops are on different lines, then a transfer is required at the stop, which will be called a transport hub. $\mathrm{i}$ - the number of the initial stop of the passenger's route, $j$ - the number of the final stop of the passenger's route, $\mathrm{k}$ - the number of a possible interchange hub. There can be several numbers. We can assume that for the studied network, from any stop i, one can get to stop $\mathrm{j}$ through at least one possible interchange hub $\mathrm{k}$. Since the network topology is given, it is possible to specify all possible routes in advance from $\mathrm{i}$ to $\mathrm{j}$ through possible interchange hubs $\mathrm{k}$.

It is necessary to note the factors that reduce the average travel time of a passenger around the city due to the formation of a system of transport hubs.

1. Direct routes from $\mathrm{i}$ to $\mathrm{j}$ are replaced by interchange routes if travel time decreases.

2. Transition to TIH from one route to another is carried out faster by the value $\Delta \mathrm{t}_{\mathrm{TIH}}$.

3. New interchange routes appear from point $i$ to point $j$ with a change at the $1^{\text {th }}$ hub due to the construction of a transport hub with $\mathrm{t}_{\mathrm{ilj}}<\mathrm{t}_{\mathrm{ij}}$.

Further, the shortest paths in time from $i$ to $j$ are found using well-known algorithms [4]. Then the whole set of possible triples of numbers $(i, k, j)$ becomes known 
and the value $\mathrm{K}$ is the maximum possible number of interchange hubs.

It is obvious that rational routes with more than one change are possible. Then such routes can be specified by a sequence of numbers $(i, k, 1, \ldots r, j)$. In addition to the network graph, the main characteristics of the passenger traffic are specified:

1. The average number of passengers traveling from $i$ to $\mathrm{j}$ (for the base period), $\mathrm{c}_{\mathrm{ij}}$ - the matrix of correspondences [6].

2. Average travel time from $\mathrm{i}$ to $\mathrm{j}$ along a straight line $\mathrm{t}_{\mathrm{ij}}$.

3. Average travel time from $i$ to $j$ through the transport hub $\mathrm{k} \mathrm{t}_{\mathrm{ikj}}$.

4. Average travel time from $i$ to $j$ through interchange hubs $\mathrm{k}$ and $1 \mathrm{t}_{\mathrm{ikj}}$.

5. The total number of passenger rides during the base period $-\mathrm{N}$.

6. The total number of stops of the transport network $-\mathrm{n}$.

7. The maximum allowable volume of passengers at the $\mathrm{k}^{\text {th }}$ interchange hub for the base period is $\mathrm{D}_{\mathrm{k}}$.

8. The minimum allowable volume of passengers at the $\mathrm{k}^{\text {th }}$ interchange hub for the base period is $\mathrm{d}_{\mathrm{k}}$.

All studied characteristics are obtained using statistical surveys, using prediction of future values.

All stops of the city network are numbered so that any stop of any line has its own unique number.

It is necessary to formulate the problem of optimal selection of the locations of TIH based on the condition of a given passenger flow and in order to reduce the time spent on the transfer of all passengers in the system of urban public transport. Further, we consider a possible mathematical formulation of the optimization problem according to the criterion T. For this, it is necessary to formulate the objective function and the limitations of this problem. A change in the value of $\mathrm{T}$ under the above mentioned assumptions can be carried out only by the choice of a TIH. The values $\mathrm{x}_{\mathrm{ikj}}$ and $\mathrm{x}_{\mathrm{iklj}}$ are considered as controlled variables, which are equal to 1 if we go from $\mathrm{i}$ to $\mathrm{j}$ through the hub $\mathrm{k}$ or $\mathrm{kl}$, and are equal to 0 otherwise.

If there is a direct path from $i$ to $j$, then it is considered to be identical, $\mathrm{x}_{\mathrm{ij}}=1$ for any $\mathrm{i}$ and $\mathrm{j}$. It is important to note that the considered model assumes that there is a rational path from $i$ to $j$, which lies on the same line, but the ride can be performed through interchange hubs $\mathrm{k}$ or $\mathrm{kl}$, since $\mathrm{t}_{\mathrm{ikj}}<\mathrm{t}_{\mathrm{ij}}$ (for example, if it is possible to use a faster transport ).

The average travel time of passengers around the city can be expressed as follows:

$$
\begin{aligned}
& \bar{T}=\frac{1}{N n^{2}}\left(\sum_{i=1}^{n} \sum_{j=1}^{n} c_{i j} t_{i j} x_{i j}+\frac{1}{K} \sum_{k}^{K} \sum_{i=1}^{n} \sum_{j=1}^{n} c_{i k j} t_{i k j} x_{i k j}+\right. \\
& \left.+\frac{1}{K L} \sum_{k}^{K} \sum_{l}^{L} \sum_{i=1}^{n} \sum_{j=1}^{n} c_{i k l j} t_{i k l j} x_{i k l j}+\ldots\right) \rightarrow \min
\end{aligned}
$$

Here, it is taken into account that each time $t_{i j}$ is calculated by the number of passengers who spend this time. From the above mentioned formula, it can be seen that more than two transfers can be taken into account. Next, the restrictions on the choice of variables $x_{i j}, x_{i k j}$, $\mathrm{X}_{\mathrm{iklj}}$ should be formulated.

The transportation from $\mathrm{i}$ to $\mathrm{j}$ with one transfer is carried out only through one selected interchange hub

$\sum_{k}^{K} x_{i k j}=1, \forall i, j ;(i \neq j)$

The transportation from $i$ to $j$ with two transfers is carried out only through one selected pair of transport hubs

$\sum_{k, l}^{K} x_{i k l j}=1, \forall i, j ;(i \neq j)$

The volume of the $\mathrm{k}^{\text {th }}$ interchange hub (out of all possible $\mathrm{K}$ hubs) is limited by the value $\mathrm{D}_{\mathrm{k}}$

$$
\begin{aligned}
& \sum_{i=1}^{n} c_{i k} x_{i k}+\sum_{j=1}^{n} c_{k j} x_{k j}+\sum_{i=1}^{n} \sum_{j=1}^{n} c_{i k j} x_{i k j}+ \\
& +\sum_{i=1}^{n} \sum_{j=1}^{n} c_{i k l j} x_{i k l j} \leq D_{k}, \forall k=(1, K) .
\end{aligned}
$$

In the problem (6), passengers leaving $\mathrm{k}$, arriving at $\mathrm{k}$ and transferring to $\mathrm{k}$ are taken into account. On the other hand, there may be a limitation on the fact that the transport hub must be large enough, for example, to cover the cost of additional construction of the hub infrastructure. Therefore:

$$
\begin{aligned}
& \sum_{i=1}^{n} c_{i k} x_{i k}+\sum_{j=1}^{n} c_{k j} x_{k j}+\sum_{i=1}^{n} \sum_{j=1}^{n} c_{i k j} x_{i k j}+ \\
& +\sum_{i=1}^{n} \sum_{j=1}^{n} c_{i k l j} x_{i k l j} \geq d_{k}, \forall k=(1, K)
\end{aligned}
$$

We get the problem (3-7) of linear Boolean programming, which, in principle, can be solved using standard computer programs $[4,7]$. In the algorithm for the solution of this problem (3-7), various options of transport hubs among the allowable ones will be sorted out, but since the objective function is minimized, the optimal transportation option will use the allowable number of interchange hubs from the condition of minimizing the objective function (3). Therefore, in this statement, the question of the optimal number of transport hubs $\mathrm{K}^{*}$ is solved automatically.

The number of routes passing through the interchange $\mathrm{k}$ (value $\mathrm{b}_{\mathrm{k}}$ ) is determined.

Let the solution of problem (3-7) be found. These are arrays of zeros and ones $\left(\mathrm{x}_{\mathrm{ikj}}, \mathrm{x}_{\mathrm{iklj}}\right)^{*}$ (asterisk marks all values obtained as a result of the solution of problem (3-7)). Then

$b_{k}=\sum_{i=1}^{n} \sum_{j=1}^{n}\left(x_{i k j}\right)+\sum_{i=1}^{n} \sum_{j=1}^{n}\left(x_{i k l j}\right),(k=1, K)$

The vector $b_{k}$ gives all the necessary information about the solution of the initially posed problem. First, 
the number of transport hubs in the solution is determined by the number of nonzero components of this vector

$$
K^{*}=\sum_{k}^{K} \delta\left(b_{k}\right),
$$

where $\delta\left(b_{k}\right)=1$, if $b_{k}>0$ and $\delta\left(b_{k}\right)=0$, if $b_{k}=0$.

We obtain the capacity of interchange hubs $B_{k}$ (the total number of passengers who visited the $\mathrm{k}^{\text {th }}$ interchange hub) using the following expressions:

$$
B_{k}=b_{k}=\sum_{i=1}^{n} \sum_{j=1}^{n} c_{i k j}\left(x_{i k j}\right)+\sum_{i=1}^{n} \sum_{j=1}^{n} c_{i k l j}\left(x_{i k l j}\right),(k=1, K)
$$

$B_{k}$ shows the number of passengers transferring at the $\mathrm{k}^{\text {th }}$ hub.

Further, the complexity of the solution of the problem (3-7) and the ways of its simplification are estimated. Considering this problem, it is believed that only single transfers are possible (with the increase in the number of transfers, the complexity of the solution increases dramatically). In this problem, only the variables $\mathrm{x}_{\mathrm{ikj}}$ are varied. Their number is determined by the number of encountered connecting routes.

The considered problem of the optimal choice of the TIH system is a multidimensional problem of linear Boolean programming and, therefore, can be solved only approximately by one of the enumeration methods. It is necessary to propose an approach that allows simplifying the solution of this problem and obtain practical options for its solution.

\section{Purpose of the Study}

The purpose of the work is to form a system of TIHs based on the optimal choice of TIH locations in urban transport network.

\section{Research Methods}

In order to solve the problem of the choice of $\mathrm{TIH}$ locations, an approach based on the definition of "effective interchange hubs" is proposed. Such hubs are candidates for the role of transport hubs.

In efficient interchange hubs, passengers are transferred in case of their travel by the fastest routes, which are determined using graphical analytical methods.

Further, using mathematical methods for the solution of integer programming problems (the solution of the knapsack problem, the branch-and-bound method), the locations of the transport hubs are determined.

Thus, the problem of the optimal choice of locations for urban transport hubs is solved in two stages.

1. At the first stage, for each transfer route, there are transport hubs $r$ that provide the shortest path from $i$ to $j$ through the transport hub $\mathrm{r}$

$$
r=\underset{k}{\arg \min }\left(t_{i k j}\right)
$$

It is necessary to note that in the future, the expression for one transfer will be denoted by the number $\mathrm{k}$, although $\mathrm{k}$ can be considered a vector of transfers, and then (11) is also valid for the case of many transfers.

The hubs of the urban transport network that satisfy the problem (11) are efficient interchange hubs. It is obvious that now only effective interchange hubs should be considered as allowable for the solution of the problem of the choice of TIH hubs. Processing this stage radically narrows the range of feasible solutions and simplifies the algorithms for the solution of the problem. A set of effective interchange hubs is found $\mathrm{R}=$ $\left(1,2,3, \ldots, r, \ldots, N_{r}\right)$, where $r$ is the number of the effective interchange hub, $\mathrm{N}_{\mathrm{r}}$ is the total number of interchange routes. In the case of two or more transfers, the transfer vector $(\mathrm{k}, 1, \ldots \mathrm{q}, \mathrm{p})$, which has its own number, can also act as a transfer.

2. At the second stage, a Boolean variable is considered: $\mathrm{x}_{\mathrm{r}}=1$, if the $\mathrm{r}^{\text {th }}$ effective hub is TIH; $\mathrm{x}_{\mathrm{r}}=0$, otherwise. Then the set of TIHs is determined by a vector of zeros and ones of dimension $\mathrm{N}_{\mathrm{r}}$.

$\mathrm{X}=\left(\mathrm{x}_{1}, \mathrm{X}_{2}, \ldots, \mathrm{x}_{\mathrm{N}}\right), \quad \mathrm{X} \in \mathrm{R}$.

At the same time, during the choice of the optimal $\mathrm{X}^{*}$, the optimal number of TIHs in the project $\mathrm{n}$

$\sum_{r=1}^{N_{r}} x_{r}^{*}=n$

Thus, it is possible to simplify the description of the mathematical model through the introduction of the concept of effective interchange hubs and a vector that determines the solution of a much smaller dimension.

\section{Findings}

The following aspects are considered as the initial data for the studied problem of finding effective interchange hubs: a matrix of interstop correspondence [6, 15], a graph of the urban transport network indicating routes, stops and possible interchange hubs, travel times between all public transport stops on all city routes [7].

The search of efficient transport hubs is carried out according to the following algorithm. A table of stops and the names of these stops are created. For each route, the numbers of stops (a chain of stops $i$ and $j$ ) through which this route passes are recorded. If a bus / trolleybus / tram follows the same route in the forward and backward directions, it is recorded as a sequence of stop numbers in the same direction. If the route is circular, then it is recorded as a sequence of stop numbers recorded twice in order to accurately determine the length of the route, the time and the number of stops that should be traveled when traveling from $\mathrm{i}$ to $\mathrm{j}$. After that, there is a list of non-stop (direct) rides from $\mathrm{i}$ to $\mathrm{j}$.

Then we consider rides from $i$ to $j$ for which there is no direct route or it exists, but the revealed connecting route will take less time. These interchange hubs are called "efficient" interchange hubs. Next, we consider rides from $\mathrm{i}$ to $\mathrm{j}$ with two interchange hubs. These are 
routes for which there is no one-transfer route, or routes that are faster than routes with one-transfer route.

For the practical implementation of the above mentioned calculation algorithm, a computer program "Efficient Transfers" is developed, which allows obtaining many effective interchange hubs for the network of urban passenger transport [7].

The correspondence matrix $\mathrm{C}^{0}=\left\{\mathrm{c}^{0}(\mathrm{i}, \mathrm{j})\right\}$ determines the average predicted value of the passenger traffic moving from stop $\mathrm{i}$ to stop $\mathrm{j}$ over a certain period of time. This time includes the standard waiting time for public transport. For routes with transfers, $c_{i k j}$ is the number of passengers traveling from $i$ to $j$ and changing at hub $\mathrm{k}$. The number of passengers who follow from $\mathrm{i}$ to $\mathrm{j}$ with two or more transfers $c_{\mathrm{ikl}} \ldots \mathrm{pj}$. . It is considered that $\mathrm{k}$ is the set of numbers of transport hubs. It is necessary to determine the largest efficient transport hubs in terms of the number of passengers. Some of these hubs will become TIHs in the future.

The creation of TIH is aimed at the improvement of the convenience of passenger service during transfer and requires significant costs for redevelopment of the transport hub area, construction of convenient crossings and additional infrastructure (commercial areas, waiting rooms, etc.). The development of a TIH system should entail a reduction in the time spent by passengers on total service. The choice of transport and interchange hubs is primarily related to the number of passengers who transfer, since the main criterion for the optimal urban transport network requires minimizing the average time of one ride.

The average time of one ride with a transfer can be expressed as:

$$
T=\frac{1}{\sum_{i, k, j} c_{i k j}} \sum_{i, j} c_{i k j} t_{i k j} \rightarrow \min
$$

where $c_{i k j}$-the number of passengers traveling from $i$ to $\mathrm{j}$, transferring to $\mathrm{k}$;

$t_{i k j}$-the time spent on passengers transfer from stop $i$ to stop $\mathrm{j}$ through $\mathrm{k}$.

In the problem (13), it is noted that for all $i$ and $j$ the transfer at the transport hub $\mathrm{k}$ is fixed. Based on it (13), we can conclude that the average time of one ride is determined by significant passenger traffic. That is, the more passengers will be processed through the formed system of transport and transport hubs, the greater the obtained reduction in time.

Having determined the set of effective transport hubs (11), it is possible to calculate the amount of passenger traffic at each hub $c_{\mathrm{r}}$.

$c_{r}=\sum_{i, j} c_{i, r, j}+\sum_{i} c_{i r}+\sum_{j} c_{r j} \quad, r=1,2, \ldots R$

where $\sum_{i, j} c_{i, r, j}$ - the number of passengers changing at the $\mathrm{r}^{\text {th }}$ interchange hub (TIH);

$\sum_{i} c_{i r}-$ the number of passengers arriving at TIH; $\sum_{j} c_{r j}$ - the number of passengers leaving TIH.

Since formula (13) expresses the criterion of the average time of one transfer ride and its values $c_{k}$ are the frequency of occurrence of the values of the time of one ride $t_{k}$, it turns out that the values of $c_{r}$ determine the main criterion for the creation of transport hubs. The amount of passenger traffic at each efficient hub provides information on where to set up TIHs.

\section{Conclusion}

Thus, the model of the optimal choice of the TIH system considered in this paper in the form of a linear Boolean programming problem and the approach based on the choice of efficient interchange hubs allow solving the problem of the optimal placement of TIHs in the urban transport network. Efficient interchange hubs are hubs in the network of urban public transport where passengers are transferred when traveling around the city, while choosing the optimal route based on the criterion of the minimum travel time. These very hubs lie at the intersection of several routes and determine the location of urban transport and interchange hubs.

The results of this research can be used in programs and projects for the development of urban transport systems in the determination of basic requirements for the formation of transport hubs.

\section{References}

1. B. Fan, Y. Yang, L. Li. Integrated optimization of urban agglomeration passenger transport hub location and network design. URASIP J. on Wirel. Communicat. and Network., 168 (2018)

2. N. Kalyuzhny, L. Losin. A method of mathematical modeling for transport hub establishment in Saint Petersburg. Transport. Res. Proc., 36, 245-251 (2018)

3. N.A. Kalyuzhny. Justification of the priority of the location of transport interchange hubs in the structure of the agglomeration by the method of mathematical modeling. Bull. of Civil Eng., 5(64), 142-148 (2017)

4. V.G. Karmanov. Mathematical Programming (Science, Moscow, 1986), 288 p

5. P. Kristersson. The role of public transport interchanges in regional planning. Reg. Magaz., 285(1), 16-17 (2012)

6. S.A. Leonova. On the formation of a system of transport interchange hubs in the Samara city district. Railway transp., 8, 58-61 (2019)

7. S.A. Leonova. Choice of locations for transport hubs of the urban passenger transport network. Transp. of the Urals, 4(63), 101-105 (2019)

8. Z.-C. Li, W. Lam, S.C. Wong. Modeling intermodal equilibrium for bimodal transportation system design problems in a linear monocentric city. Transport. Res., 46, Part B (2012) 
9. I. Ludan, E. Maiorov, J.D.M. Santana, O. Saprykin. Integrated approach to building a microscopic city model. In Proceedings of the 5th International Young Scientists Conference on Information Technologies, Telecommunications and Control Systems (2018), p. 8

10. A. Monzón, S. Hernández, F. Di Ciommo. Efficient urban interchanges: the City-HUB model. Transport. Res. Proc., 14, 1124-1133 (2016)

11. A.A. Shagimuratova System analysis in determining the priority directions for the development of transport hubs of railway transport. Urban plan., 2(42), 63-71 (2016)

12. E.V. Sherbina, N.V. Danilina, D.N. Vlasov. City planning issues for sustainable development. Int. J. of Appl. Eng. Res., 10(22), 43131-43138 (2015)

13. C. Sun, X. Chen, H M. Zhang, Z. Huang. An Evaluation Method of Urban Public Transport Facilities Resource Supply Based on Accessibility. Hindawi J. of Advan. Transport., 11, (2018)

14. D.N. Vlasov. Transport hubs. Monograph, 2nd ed. (MSSU, Moscow, 2017), $192 \mathrm{p}$

15. M.R. Yakimov, A.A. Arepyeva. Transport planning. Features of modeling traffic flows in large Russian cities. Monograph (Logos, Moscow, 2016), 280 p 